\title{
Triple grouping and period-three oscillations in minority-game dynamics
}

\author{
Jia-Qi Dong, ${ }^{1}$ Zi-Gang Huang,,${ }^{1,2, *}$ Liang Huang, ${ }^{1,2}$ and Ying-Cheng Lai ${ }^{2,3}$ \\ ${ }^{1}$ Institute of Computational Physics and Complex Systems, Key Laboratory for Magnetism and Magnetic Materials \\ of the Ministry of Education, Lanzhou University, Lanzhou 730000, China \\ ${ }^{2}$ School of Electrical, Computer and Energy Engineering, Arizona State University, Tempe, Arizona 85287, USA \\ ${ }^{3}$ Department of Physics, Arizona State University, Tempe, Arizona 85287, USA
}

(Received 22 June 2014; revised manuscript received 21 October 2014; published 23 December 2014)

\begin{abstract}
Dynamical systems based on the minority game (MG) have been a paradigm for gaining significant insights into a variety of social and biological behaviors. Recently, a grouping phenomenon has been unveiled in MG systems of multiple resources (strategies) in which the strategies spontaneously break into an even number of groups, each exhibiting an identical oscillation pattern in the attendance of game players. Here we report our finding of spontaneous breakup of resources into three groups, each exhibiting period-three oscillations. An analysis is developed to understand the emergence of the striking phenomenon of triple grouping and period-three oscillations. In the presence of random disturbances, the triple-group/period-three state becomes transient, and we obtain explicit formula for the average transient lifetime using two methods of approximation. Our finding indicates that, period-three oscillation, regarded as one of the most fundamental behaviors in smooth nonlinear dynamical systems, can also occur in much more complex, evolutionary-game dynamical systems. Our result also provides a plausible insight for the occurrence of triple grouping observed, for example, in the U.S. housing market.
\end{abstract}

DOI: 10.1103/PhysRevE.90.062917

PACS number(s): 89.75.Fb, 05.10.Ln, 89.65.-s, 89.75.Hc

\section{INTRODUCTION}

A paradigmatic model to study a variety of complex social and biological behaviors is the minority game (MG) [1-4]. In the simplest setting of multiple identical agents with two resources, e.g., the El Farol bar problem in game theory [1], each agent has two strategies to choose from: either exploiting the resource (going to the bar) or no (staying at home). In this case, the winning strategy is the one taken by the minority of agents [3]. In the MG system, in spite of each agent's best effort to choose the minority strategy, there is no guarantee that the strategy chosen would indeed be the minority one. Often, coherence or herd behavior, in which a vast majority of the agents choose an identical strategy, can emerge. This can cause large oscillations in the usage of the available resource, leading to low efficiency for the system. In the past two decades, there has been a great deal of interest in MG dynamics [5-25]. In physics, MG dynamics has been shown to be highly relevant to problems associated with nonequilibrium phase transitions [26-28].

In a previous work [29], we studied the MG dynamics with multiple resources in which agents are homogeneous and the game rules are uniform, where at each time step an individual agent has to choose one of the $k(k>2)$ resources or strategies based on local information only. We found the phenomenon of grouping, in which the resources spontaneously break into two groups, with resources in the same group having same number of attendees. Intuitively, this phenomenon can be explained using a variant of the El Farol bar problem, in which there are $k$ bars available. Every night each individual can choose to go to any of the bars based on information about the bar attendances in the previous night. The number of individuals attending any specific bar thus varies from night to night, and

*huangzg@1zu.edu.cn typically exhibits a kind of oscillation with time. Depending on the probability that each agent selects a less crowded bar, two characteristically distinct oscillating patterns of the bar attendance can emerge, where a subset of bars exhibit one pattern while the remaining bars belong to the other camp. It was suggested [29] that this grouping mechanism may be responsible for the phenomenon of spontaneous grouping of stocks observed in real financial markets.

In this paper, we report a period-three grouping behavior in multiple resource MG systems. In such a case, the available resources in the system spontaneously break into three groups, in which the number of agents attending each group exhibits period-three oscillations, and the oscillation patterns of different groups are $2 \pi / 3$ phase shifted with respect to each other. We derive an analytic theory to understand the period-three phenomenon in MG systems. We also study the bifurcations to oscillations of higher periods and the basis of attractions of the distinct states. Considering that the period-three behavior is fundamental in smooth nonlinear dynamical systems [30], our finding that oscillations of odd periods can occur in agent-based MG systems is striking. This may have practical relevance as triple grouping has been observed in real economical systems, e.g., the U.S. housing market [31], and our result represents a plausible insight for this behavior. In the presence of random fluctuations, a period-three grouping state becomes transient, and we provide a scaling analysis of its average lifetime using two levels of approximation. We also provide a theoretical argument that periodic grouping states of higher periods are increasingly unlikely as the period becomes higher, suggesting that such periodic states should rarely appear in real-world systems.

This work uncovers the period-three behavior, a fundamental phenomenon in traditional nonlinear dynamics, in MG dynamics. The finding can potentially be interesting to workers in both fields. Nonetheless, whether the period-three behavior can have direct impact on human-subject strategy evolution 
remains an open issue. For example, suppose each player can obtain global information about the game dynamics. When a regular oscillating behavior arises, the players will exploit the regularity spontaneously, thereby destroying the oscillations. In real-world situations most individuals are unable to get access to global information and thus may not be aware of any regular oscillatory dynamics of the system in time to respond to it. In this case, we expect the period-three behavior uncovered in this work to be relevant, as evidenced by the phenomenon of triple grouping observed in the U.S. housing market.

In Sec. II we describe our multiple resource MG dynamics model. In Sec. III we present theoretical analysis and numerical results with respect to the following three issues: the emergence of period-three grouping states, the transient nature of such states and the associated scaling behavior, and the rarity of periodic grouping states of higher periods. In Sec. IV we offer conclusions and discussions.

\section{MULTIRESOURCE MG-DYNAMICS MODEL}

The commonly studied setting of the MG model [1-3,5-25] has two resources (e.g., the bar and home in the El Farol bar problem). At each time step, every agent can choose one of the two strategies, e.g., to attend the bar or stay at home. To make a decision, an agent would rely on historical information of bar attendance. The El Farol bar problem can be generalized to MG dynamics with multiple resources, as follows.

Consider an MG system of $N$ interacting agents competing for $k(k>2)$ available resources/strategies, labeled by $s$, where $s=1,2, \ldots, k$. Each resource has a limited capacity, so the number of agents it can accommodate has the upper bound $n_{c}=N / k$. At each round of game (or equivalently, each time step), each agent chooses one from the $k$ available resources. Let $n_{s}$ be the attendance of a particular resource $s$, i.e., the number of agents selecting $s$. If $n_{s} \leqslant n_{c}, s$ is a minority strategy, so the agents selecting strategy $s$ win the game. However, if $n_{s}>n_{c}$, resource $s$ is too crowded so that the strategy fails and the agents taking it lose the game. An optimal solution (Nash equilibrium) of the system is thus $n_{s}=n_{c}$.

In a realistic situation, agents gain information about the dynamical state of the whole system from other agents. It is in this sense that the agents interact with each other. At each time step, with probability $p$ each agent selects one of the $k$ available resources based on local information, i.e., information from neighboring agents. In Ref. [29], $p$ is called the minority-preference probability. The probability that an agent acts randomly is $1-p$. When agent $i$ makes an informed decision based on interactions with its $d$ neighbors in the network, the required information is all its neighbors' strategies and, among them, the winners of the game, i.e., those neighboring agents who chose the winning resources at the last time step. Let $\Pi=\left\{s_{\text {win }}\right\}$ be the set of resources selected by $i$ 's winning neighbors, where a resource may appear a number of times if it has been chosen by different winning neighbors. With probability $p$, agent $i$ chooses one winning resource randomly from the set $\Pi$. Thus, the probability $P_{s}$ for one given resource $s$ to be selected is proportional to the times it appears in $\Pi$ (denoted by $\left.\eta_{s}\right)$. We have $P_{s}=\eta_{s} / \operatorname{Card}(\Pi)$, where $\operatorname{Card}(\Pi)$ is the number of elements in $\Pi$. Here the decision making of each agent is dependent upon the outcome of the game at the previous step as it adopts the winners' choices based on local information. The higher probability for agents to keep the choices won in the last round can be considered as a conditional response behavior [32] in the multiagent interaction setting. If the winning-resource set $\Pi$ is empty, agent $i$ will randomly select one from the $k$ available resources. For the case where an agent acts without any local information (with probability $1-p$ ), it will choose a resource randomly from the $k$ available ones.

\section{RESULTS AND ANALYSIS}

For the case that the $k$ resources are organized into $M$ groups, we denote the number of resources in group $i$ to be $k_{g_{i}}$ with $i \in\{1,2, \ldots, M\}$, and denote the number of all agents choosing the resources in group $i$ to be $N_{g_{i}}$, where $k=\sum_{i} k_{g_{i}}$ and $N=\sum_{i} N_{g_{i}}$. In the stable state, the attendances of each resource in the same group $i$ are identical [29]: $n_{s} \doteq N_{g_{i}} / k_{g_{i}}$. That is, the $N_{g_{i}}$ agents in group $i$ are equally distributed among the $k_{g_{i}}$ resources.

The master equation underlying the MG dynamics can be written as

$$
\begin{aligned}
n_{s}(t+1) & =n_{\mathrm{ir}}=(1-p) N \frac{1}{k}, \quad \text { for } \quad n_{s}(t)>n_{c} \\
n_{s}(t+1) & =n_{\mathrm{ir}}+n_{\mathrm{ra}} \\
& =(1-p) N \frac{1}{k}+p N \frac{n_{s}(t)}{N_{\text {win }}}, \text { for } n_{s}(t)<n_{c},
\end{aligned}
$$

where $N_{\text {win }}$ is the total number of agents in the winning-resource group at time $t$, i.e., those that satisfy $n_{s}(t)<n_{c}$. The failing resources at time $t$, i.e., those for which $n_{s}(t)>n_{c}$ holds, may have nonzero attendance $n_{\text {ir }}$ at time $t+1$ due to agents' "irrational" selections with probability $1-p$. For a nonzero value of $p$, since $n_{\mathrm{ir}}<n_{c}$, the failing resources become winning ones at time $t+1$. For a winning resource at time $t$, its attendance at $t+1$ is composed of two contributions: one due to the $n_{\text {ir }}$ irrational agents and another due to the $n_{\text {ra }}$ rational agents, where $n_{\text {ra }}$ is proportional to $n_{s}(t)$, the number of agents taking the winning strategies at the previous time step, for the reason that agents spread the information about their winning strategies. This self-enhancing process of $n_{s}$ persists until it increases over $n_{c}$. Thus, initiated from a sufficiently small value of $n_{s}(t)$, it is possible for $n_{s}(t+1)$ to be less than $n_{c}$. That is, resource $i$ can remain to be winning for a consecutive period of time. Figure 1 shows representative periodic behaviors of period greater than 2 with consecutive winning states separated by a single failing state. For example, a period-three behavior is observed, such as $(\ldots, w, f, w, w, f, w, w, f, w, \ldots)$. Additionally, the social efficiencies of different periodic states are different due to their distinct modes of oscillation. In the following, we focus on the period-three state by using two approaches: solutions of the master equation and direct simulation of the $\mathrm{MG}$ dynamics. We then discuss periodic states of higher periods.

\section{A. Emergence of period-three state}

For the period-three state, the $k$ resources are organized into $M=3$ groups, and the attendances to the three different 

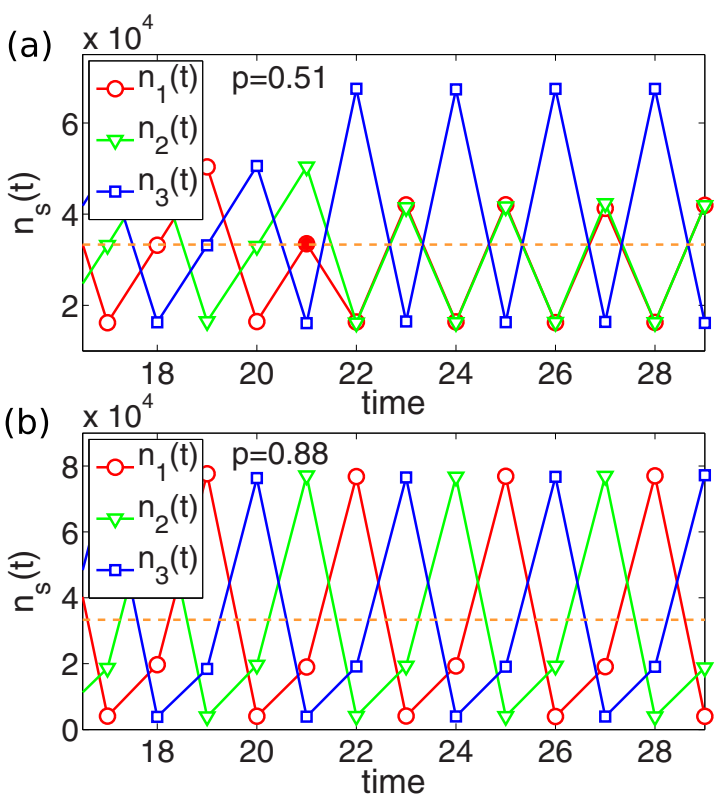

FIG. 1. (Color online) For an MG system of $k=3$ resources on fully connected network (FCN), time series $n_{s}(t)$ from simulation: (a) about a state transition from $p_{3} g_{3}$ to $p_{2} g_{2}$ for $p=0.51$, and (b) a stable $p_{3} g_{3}$ state for $p=0.88$. The system is composed of $N=10^{5}$ agents.

groups exhibit the same value but alternate in time with a $2 \pi / 3$ phase difference with respect to each other, as shown in Fig. 1(b). For convenience, we denote such period-three and group-three state by $p_{3} g_{3}$. In order for such a state to arise as the steady state, the attendance of resources in one given group must satisfy the equations

$$
\begin{aligned}
n_{s}(t) & =(1-p) N \frac{1}{k}, \\
n_{s}(t+1) & =(1-p) N \frac{1}{k}+p N \frac{n_{s}(t)}{N_{\text {win }}}, \\
n_{s}(t+2) & =(1-p) N \frac{1}{k}+p N \frac{n_{s}(t+1)}{N_{\text {win }}},
\end{aligned}
$$

with the constraints $n_{s}(t)<n_{s}(t+1)<n_{c}<n_{s}(t+2)$ and $n_{s}\left(t^{\prime}\right)=n_{s}\left(t^{\prime}+3\right)$. There are two groups composed of winning resources and one group of failing resources, and we have $N_{\text {win }}=k\left[n_{s}(t)+n_{s}(t+1)\right] / M$.

Generally, for the case that the number of resources $k$ is not an integer multiple of $M$, the $M$-group state also emerges with each group size about $k / M$, which is nonetheless less stable than the states in systems where $k$ is an integer multiple of $M$.

Period-three orbit. The period-three state can be observed for cases of more than three resources, and the required number of resources is not necessarily integer multiples of 3. For example, we have observed that a system with $k=16$ resources can self-organize into three groups of sizes $k_{1}=5, k_{2}=5$, and $k_{3}=6$, respectively. Here, to facilitate analysis, we focus on systems with $k=3$ resources (labeled by $s=1,2,3$ ), which is the minimally required number of resources that satisfies Eq. (2) with the periodic condition. In this case, each of the three groups possesses just one resource: $k_{1}=k_{2}=k_{3}=1$. The solution of Eqs. (2) is

$$
\begin{aligned}
x_{1} & \equiv n_{1}(t)=n_{3}(t+1)=n_{2}(t+2) \\
& =\frac{1}{3} N(1-p)=\left.n_{\mathrm{ir}}\right|_{M=3}, \\
x_{2} & \equiv n_{2}(t)=n_{1}(t+1)=n_{3}(t+2) \\
& =\frac{\sqrt{(1-p)(1+2 p)}}{3} N, \\
x_{3} & \equiv n_{3}(t)=n_{2}(t+1)=n_{1}(t+2) \\
& =\frac{1}{3} N(2+p)-\frac{\sqrt{(1-p)(1+2 p)}}{3} N .
\end{aligned}
$$

The existence of the $p_{3} g_{3}$ state requires $x_{1}<x_{2}<n_{c}<x_{3}$ and $N=\sum_{s} n_{s}=\sum_{i} x_{i}$. Apparently, we have $x_{1}<n_{c}$. The condition $x_{2}<n_{c}$ implies the requirement for the parameter $p: p>0.5$, where for $p \leqslant 0.5$, no $p_{3} g_{3}$ orbit can exist. Figures 2(a)-2(c) show the dynamical states of the system $\left(n_{1}, n_{2}, n_{3}\right)$ in the phase space of triangular representation. In Fig. 2(b), the orbits for the state of period two and group two (denoted by $p_{2} g_{2}$ ) correspond to the three line segments with solid triangles as vertices. The $p_{3} g_{3}$ state corresponds to the clockwise or the counterclockwise triangular orbits (blue solid
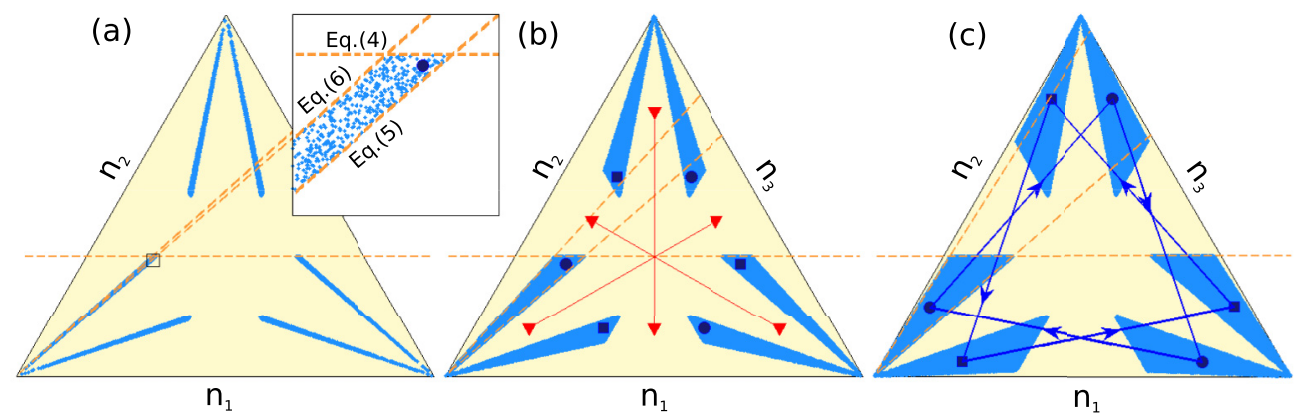

FIG. 2. (Color online) For an MG system of $k=3$ resources on fully connected networks (FCNs), basins of distinct grouping states in the triangular representation of the phase space defined by the variables $n_{1}, n_{2}$, and $n_{3}$. In each panel, the blue (dark gray) and yellow (light gray) regions denote the basins of the $p_{3} g_{3}$ and $p_{2} g_{2}$ states, respectively. The three dashed lines are the boundaries of the basins corresponding to the constraints (4)-(6), as labeled in the inset of (a). (a)-(c) Theoretically determined orbits of the system in the states $p_{2} g_{2}$ and $p_{3} g_{3}$ for $p=0.51$, 0.62 , and 0.88 , respectively. For clarity, in (b) the orbits of $p_{2} g_{2}$ are plotted by three line segments (with solid triangle as vertices), and in (c) the orbits of $p_{3} g_{3}$ are illustrated by the clockwise or counterclockwise triangular orbits (solid lines with full circles or squares as vertices). The arrowheads associated with the $p_{3} g_{3}$ orbit denote the direction of dynamical evolution. The system size is $N=10^{4}$. 
lines), as shown in Fig. 2(c), with the three respective vertices (solid circle or solid square) of the two triangles determined by the six possible permutations of $x_{1}, x_{2}$, and $x_{3}$ in Eq. (3). The corresponding time series $n_{s}(t)$ of the $p_{3} g_{3}$ and $p_{2} g_{2}$ states are shown in Figs. 1(a) and 1(b), respectively.

Basins of attraction. Figure 2 shows the basins of the two final states $p_{3} g_{3}$ and $p_{2} g_{2}$ of a fully connected MG networked system in the simplex representation of the three variables $\left(n_{1}, n_{2}, n_{3}\right)$, which are obtained from iterations of the master equations (1a) and (1b) for $10^{4}$ time steps. The basin of the $p_{3} g_{3}$ state consists of six blue (or dark gray) regions, and that of the $p_{2} g_{2}$ state is the complementary (yellow or light gray) region. If the initial state $\left(n_{1}, n_{2}, n_{3}\right)$ is chosen from any of the six blue (dark gray) regions, the system will evolve into the triangular orbit of the stable $p_{3} g_{3}$ state after a few iterations. Specifically, the clockwise triangular orbit of $p_{3} g_{3}$ results from the initial state $n_{1}\left(t_{0}\right)<n_{2}\left(t_{0}\right)<n_{c}<n_{3}\left(t_{0}\right)$ but the counterclockwise triangular orbit is from the state with transposition of $n_{2}\left(t_{0}\right)$ and $n_{3}\left(t_{0}\right)$. The system evolving from the initial state in the yellow (light gray) region will finally settle into the $p_{2} g_{2}$ state, i.e., one of the three line-segment orbits in Fig. 2(b).

The basin boundaries of the $p_{3} g_{3}$ and $p_{2} g_{2}$ marked by the three dashed lines in Fig. 2 are determined by the three constraints of the $p_{3} g_{3}$ with respect to the initial states, which can be obtained analytically. Taking the clockwise triangular orbit as an example, one constraint is that the second largest attendance in $n_{s}$ is smaller than $n_{c}$, i.e.,

$$
n_{2}\left(t_{0}\right)<n_{c} .
$$

In the opposite case of $n_{2}\left(t_{0}\right)>n_{c}$, both resources 2 and 3 are failing ones. From Eq. (1a) we have

$$
n_{2}\left(t_{0}+1\right)=n_{3}\left(t_{0}+1\right)=n_{\mathrm{ir}} .
$$

The system then transitions from the $p_{3} g_{3}$ to the $p_{2} g_{2}$ states. The constraint (4) corresponds to the basin boundary $n_{2}\left(t_{0}\right)=$ $n_{c}$, as shown by the horizontal dashed line in Fig. 2 (labeled in the inset).

The second constraint of $p_{3} g_{3}$ is that the values of $n_{s}\left(t_{0}\right)$ 's are such that the second largest variable $n_{s}$ obeys the following inequality: $n_{1}\left(t_{0}+1\right)<n_{c}$. In the opposite case of $n_{1}\left(t_{0}+\right.$ 1) $>n_{c}$, both resources 1 and 2 are failing ones. Similarly, the system transitions from the $p_{3} g_{3}$ to the $p_{2} g_{2}$ states because of $n_{1}\left(t_{0}+2\right)=n_{2}\left(t_{0}+2\right)=n_{\text {ir }}$. Specifically, from Eq. (1b), the second constraint to the state at time $t_{0}$ can be expressed by the inequality

$$
n_{1}\left(t_{0}+1\right)=n_{\text {ir }}+p N \frac{n_{1}\left(t_{0}\right)}{n_{1}\left(t_{0}\right)+n_{2}\left(t_{0}\right)}<n_{c},
$$

where $n_{\text {ir }}=(1-p) N k_{1} / k$. For the simple case of $k=3$, the solution is given by

$$
n_{2}\left(t_{0}\right)>2 n_{1}\left(t_{0}\right)
$$

indicating that the basin boundary is determined by $n_{2}\left(t_{0}\right)=$ $2 n_{1}\left(t_{0}\right)$, as marked by the dashed line in the inset of Fig. 2 (labeled in the inset).

The third constraint on $p_{3} g_{3}$ is a further requirement for condition (5); i.e., the difference between $n_{1}\left(t_{0}\right)$ and $n_{2}\left(t_{0}\right)$ should not be too large. The reason is that, if $n_{2}\left(t_{0}\right)$ is too large, then $n_{1}\left(t_{0}+1\right)$ would be too small to satisfy the constraint (5) $n_{1}\left(t_{0}+1\right)<2 n_{3}\left(t_{0}+1\right)$. This constraint to the initial state can be expressed as

$$
n_{1}\left(t_{0}+1\right)=n_{\mathrm{ir}}+p N \frac{n_{1}\left(t_{0}\right)}{n_{1}\left(t_{0}\right)+n_{2}\left(t_{0}\right)}>2 n_{\mathrm{ir}},
$$

which requires that the initial states,

$$
n_{2}\left(t_{0}\right)<\frac{(4 p-1)}{(1-p)} n_{1}\left(t_{0}\right)
$$

correspond to the third basin boundary marked by the dashed lines in Fig. 2 (labeled in the inset). It should be noted that, all the three constraints (4)-(6) concern the present second-largest resource attendance in the system. Additionally, for the three boundaries associated with (4)-(6), only the third boundary depends on the bifurcation parameter $p$. The areas of the $p_{3} g_{3}$ basins (blue or dark gray regions) increase with $p$. Since the condition for the existence of $p_{3} g_{3}$ orbits is $p>0.5$, they correspond to the nonzero area of the $p_{3} g_{3}$ basins. Furthermore, the six different blue basin regions of $p_{3} g_{3}$ correspond to the six permutations of the three resources obeying the three constraints on the initial states, respectively.

Bifurcations. Figures 3(a) and 3(c) show the theoretically obtained bifurcation diagrams from Eq. (1) with the minoritypreference probability $p$ for fully connected networks (FCNs) and regular random networks (RRNs), respectively. We see that the attendance of resources in the $p_{2} g_{2}$ state is stable in the whole parameter range considered, while the $p_{3} g_{3}$ state is unstable for $p<p_{c}=0.5$ and becomes stable afterwards. Associated with the $p_{2} g_{2}$ state, the $k$ resources break into two groups, with resources in the same group possessing identical attendance. The attendance to both groups tends to oscillate in time, but the smaller group exhibits larger oscillations. The relative attendance $n_{s}(t) / N$ associated with the $p_{2} g_{2}$ state thus consists of two pairs of lines (blue solid lines) corresponding to the respective groups (it appears that there are only three lines, but this is due to the fact that two bottom lines of the two groups are degenerate). In the $p_{3} g_{3}$ state, the three groups share the same values $x_{1}, x_{2}$, and $x_{3}$, as shown in Fig. 1(b), so there are only three distinct lines (red dashed lines) in the bifurcation diagram. For comparison, simulation results are also shown (open blue diamonds and red circles for $p_{2} g_{3}$ and $p_{3} g_{3}$, respectively). From Fig. 3(a), we see that the simulation results for the FCN structure agree well with the theoretical result from the master equations Eq. (1). However, the simulation results for the RRN structure do not agree well with the predictions of Eq. (1). We note that the RRN structure is sparse and homogeneous, in which each agent interacts with a finite number $d$ of neighbors (the FCN structure has $d \rightarrow N)$. In fact, the RRN model is more realistic as an agent cannot obtain global information about the strategies that all other agents took. It is necessary to modify the simple master-equation theory for sparse network systems.

To develop a theory to predict the grouping structure for random networks, we note that a failed agent may be surrounded by agents from the same group (who will likewise fail the game). In this case, the failed agent has no minority strategy to imitate because the set $\Pi$ is empty and thus will randomly select one of the $k$ available resources. The master 

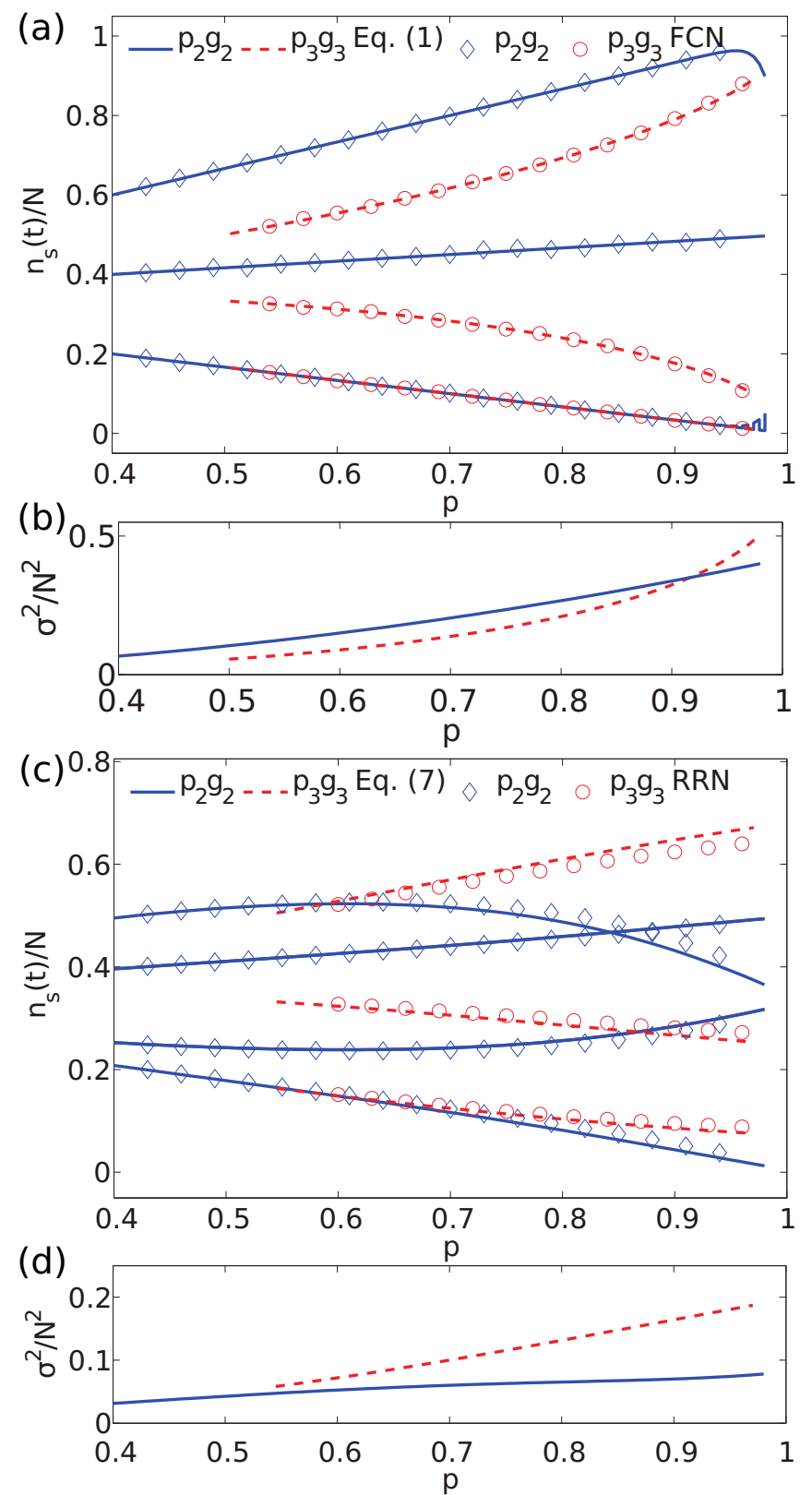

FIG. 3. (Color online) Bifurcation diagram and the corresponding social efficiency with minority-preference probability $p$ leading to the $p_{2} g_{2}$ and $p_{3} g_{3}$ states. (a),(b) Theoretical results from Eq. (1) and simulation results for fully connected networks; (c),(d) theoretical results from Eq. (7) and simulation results for random networks with $d=4$. The system has $N=2.5 \times 10^{5}$ agents and $k=3$ resources. All simulation results are obtained for 200 time steps for both $p_{3} g_{3}$ and $p_{2} g_{2}$ states. The region for $p_{3} g_{3}$ from simulation cannot reach the theoretical threshold $p_{c}=0.5$, because the state is only marginally stable in the vicinity of $p_{c}$.

equation (1) should then be modified as

$$
\begin{aligned}
n_{s}(t+1)= & (1-p) \frac{N}{k}+N p\left[\frac{n_{s}(t)}{N_{\text {win }}}-\frac{k-1}{k}\left(1-\frac{N_{\text {win }}}{N}\right)^{d}\right], \\
& \text { for } n_{s}(t)<n_{c},
\end{aligned}
$$

$$
\begin{aligned}
n_{s}(t+1)= & (1-p) \frac{N}{k}+N p\left[\left(1-\frac{N_{\text {win }}}{N}\right)^{d} \frac{1}{k}\right], \\
& \text { for } n_{s}(t)>n_{c} .
\end{aligned}
$$

Predictions from this modified master-equation set fit well with the simulation results' RRNs, as shown in Fig. 3(c). The social efficiency of the system is dependent upon the oscillatory behavior of $n_{s}(t)$ and can be measured by the variance $\sigma^{2}=\sum_{s}\left\langle\left(n_{s}(t)-N / k\right)^{2}\right\rangle_{t}$, where a larger value of $\sigma^{2}$ corresponds to lower efficiency. Figures 3(b) and 3(d) show the normalized variance $\sigma^{2} / N^{2}$. We see that the social efficiency decreases monotonously with $p$, and the two different periodic states exhibit distinct efficiencies.

\section{B. Transient dynamics of period-three grouping states}

MG dynamics in real systems are typically stochastic processes due to intrinsic randomness and various kinds of external disturbances. When stochastic effects are present, the system wanders randomly about the $p_{3} g_{3}$ state, as determined by Eq. (3). The period-three state then becomes transient with finite lifetime. Figure 4 shows the expected lifetime $T$ of the $p_{3} g_{3}$ state from simulation for $p \approx p_{c}=0.5$ for different system sizes. We see that, as $\delta p=p-p_{c}$ is increased, the lifetime increases exponentially. To develop an analytic understanding of the transient behavior of the period-three state in a concrete setting, we assume that initially the system is located at the orbit point $\left(x_{1}, x_{2}, x_{3}\right)$. The transient dynamics of orbits of higher periods can be developed accordingly.

First-order approximation. Due to intrinsic randomness, the state variables of the system, $\left[n_{1}(t), n_{2}(t), n_{3}(t)\right]$, fluctuate

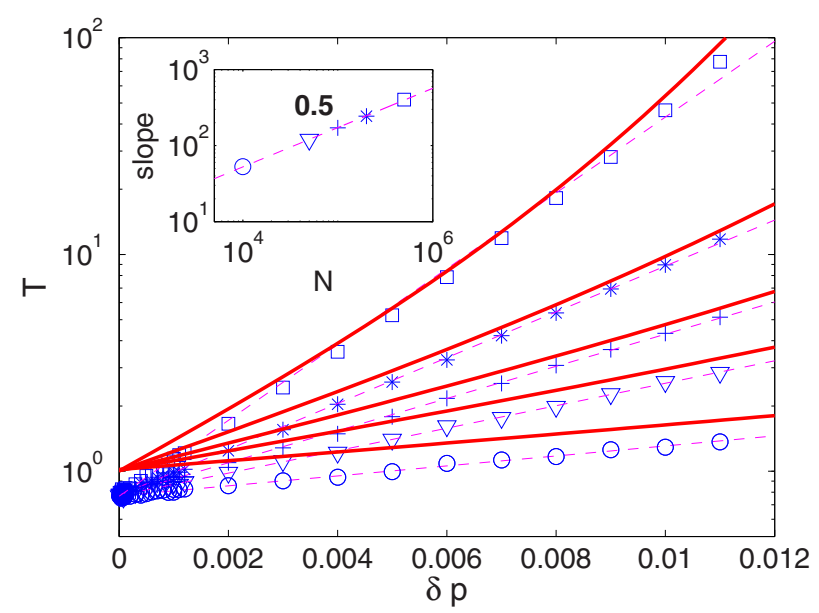

FIG. 4. (Color online) Expected lifetime $T$ of the period-three state $p_{3} g_{3}$ in the vicinity of $p_{c}=0.5$ obtained from simulation (blue/dark gray symbols) and from Eq. (11) (solid curves). All systems have $k=3$ resources and their sizes are $N=10^{4}$ (circles), $5 \times 10^{4}$ (triangles), $10^{5}$ (crosses), $2 \times 10^{5}$ (stars), and $5 \times 10^{5}$ (squares). The dashed lines are respective linear fittings to the simulation results. As indicated in the inset, the slope of the linear fitting, or the exponent of exponential increase of $T$ with $\delta p=p-p_{c}$, scales as $\sim \sqrt{N}$. 
about their respective expected values. If the system is at the orbit point $\left(x_{1}, x_{2}, x_{3}\right)$ at time $t-1$, the mechanism for the disappearance of the period-three state $p_{3} g_{3}$ at $t$ is $n_{1}(t)>n_{c}$, i.e., the present second largest value of $n_{s}$ violates the first constraint (4). The probability for each individual agent to choose resource 1 at $t$ is

$$
f_{t}=(1-p) \frac{1}{k}+p \frac{x_{1}}{x_{1}+x_{2}} .
$$

The variable $n_{1}(t)$ thus obeys the binomial distribution determined by $f_{t}$ as

$$
P\left[n_{1}(t)\right]=\mathfrak{B}[z ; Z, f] \equiv\left(\begin{array}{l}
Z \\
z
\end{array}\right) f^{z}(1-f)^{Z-z},
$$

where $z=n_{1}(t), Z=N$, and $f=f_{t}$. The relative standard deviation (RSD) is given by

$$
\sigma / \bar{n}_{1}=\sqrt{N f_{t}\left(1-f_{t}\right)} /\left(N f_{t}\right)=\sqrt{\left(1-f_{t}\right) /\left(N f_{t}\right)},
$$

where $\bar{n}_{1}$ is the expected value of $n_{1}$. The probability for $n_{1}(t) \leqslant n_{c}$, i.e., the probability for the period-three state $p_{3} g_{3}$ to survive, is

$$
P_{\text {live }}=\sum_{n_{1} \leqslant n_{c}} P\left[n_{1}(t)\right]
$$

so $P_{\text {death }}=1-P_{\text {live }}$ is the probability for the period-three state to disappear. When this occurs, there is a transition from $p_{3} g_{3}$ to $p_{2} g_{2}$ states within one time step if the system is initially located at $\left(x_{1}, x_{2}, x_{3}\right)$. As $p$ is increased, $P_{\text {death }}$ decreases for the reasons that both the expected value of $n_{1}$ (here is just $x_{2}$ ) and the RSD of $n_{1}$ decreases.

The lifetime $\tau$ of the period-three state $p_{3} g_{3}$ can be approximated by $P_{\text {live }}$. The analysis leading to Eq. (10) indicates that the disappearance of $p_{3} g_{3}$ is due to violation of the constraint (4) in one time step. In this case, the lifetime of $p_{3} g_{3}$ is $\tau=0$. To analyze cases of longer lifetime, we use a first-order approximation to neglect the accumulated effect of fluctuations and assume that the system is located exactly at the vertices of the orbit provided that it is still in the basin of $p_{3} g_{3}$. The expected lifetime of $p_{3} g_{3}$ is then given by

$$
T=\sum_{\tau=0}^{\infty} \tau\left(P_{\text {live }}\right)^{\tau}\left(1-P_{\text {live }}\right)=\frac{P_{\text {live }}}{1-P_{\text {live }}} .
$$

Figure 4 compares the prediction of Eq. (11) with simulation results. We see that our analysis based on the first-order approximation captures the exponential behavior of the lifetime with $p$. Considering that the long-term effect of random fluctuations may accumulate and induce the disappearance of the period-three state, the lifetime given by Eq. (11) is thus an overestimate.

Second-order approximation. To obtain a more accurate estimate of the transient lifetime of period-three state, the accumulated effect of intrinsic fluctuations must be included. Here we use a second-order approximation that takes into account one-step accumulation of fluctuations. As can be seen from our analysis of basins of attraction, violation of constraint (5) or (6) at the present time induces "death" of the $p_{3} g_{3}$ at the next or the following time steps. One-step accumulation of fluctuation corresponds to the first case, where the system deviates from constraint (5) but constraint (4) is still satisfied at $t$. In this case, the period-three state disappears at time $t+1$. The probability of death of $p_{3} g_{3}$ can then be estimated.

Suppose the system is in state $\left(x_{1}, x_{2}, x_{3}\right)$ at time $t-1$. If the system is still in the period-three state at time $t+1$, $n_{3}$ would represent the second largest attendance. From the distribution of $n_{3}(t+1)$, we can calculate the probability of disappearance of the period-three state. Specifically, the probability for each agent to choose resource 3 at time $t+1$ is

$$
f_{t+1}=(1-p) \frac{1}{k}+p \frac{n_{3}(t)}{n_{3}(t)+n_{1}(t)} .
$$

The distribution of $n_{3}(t+1)$ is binomial with parameter $f_{t+1}$ :

$$
P\left[n_{3}(t+1)\right]=\mathfrak{B}\left[n_{3}(t+1) ; N, f_{t+1}\right] .
$$

Differing from Eqs. (8) and (9) under the first-order approximation, here the distribution of the second largest attendance is determined by the previous state rather than the relocated orbit point $\left(x_{1}, x_{2}, x_{3}\right)$ because fluctuations at time $t$ have been taken into account. The probability for $p_{3} g_{3}$ to sustain for two continuous time steps, starting from $\left(x_{1}, x_{2}, x_{3}\right)$, is

$$
P_{\text {live }}^{(2)} \equiv \sum_{n_{3}(t+1) \leqslant n_{c}} \sum_{n_{1}(t) \leqslant n_{c}} P\left[n_{3}(t+1) \mid n_{1}(t)\right] P\left[n_{1}(t)\right] .
$$

Then the expected lifetime of the $p_{3} g_{3}$ state is

$$
T=\sum_{\tau=1}^{\infty} \tau\left(\sqrt{P_{\text {live }}^{(2)}}\right)^{\tau}\left(1-\sqrt{P_{\text {live }}^{(2)}}\right)=\frac{\sqrt{P_{\text {live }}^{(2)}}}{1-\sqrt{P_{\text {live }}^{(2)}}},
$$

which is similar in form to Eq. (11) from the first-order approximation. The numerical result from Eq. (12) diverges rapidly as $\delta p$ is increased, thus is not shown in Fig. 4. However, the result is more accurate as compared with Eq. (11) in the small $\delta p$ region.

\section{Periodic grouping states of higher periods}

To be concrete, we address the existence of $p_{k} g_{k}$ state for MG systems with $k$ resources. Similar to Eq. (3) for $p_{3} g_{3}$ state for systems of $k=3$ resources, the attendances $n_{s}$ of the $k$ resources in the $p_{k} g_{k}$ state satisfy $x_{1}<x_{2}<\cdots<x_{k}$, where $x_{k}>n_{c}$. Temporally, each resource undergoes $k-1$ consecutive winning states with $n_{s}=x_{1}, \ldots, x_{k-1}$, separated by a single failing state with $n_{s}=x_{k}$. From Eq. (1b), we have that the difference $\delta x_{i} \equiv x_{i+1}-x_{i}=q \delta x_{i-1}$ is a geometric series with ratio $q=p N / N_{\text {win }}$. We thus have

$$
\begin{aligned}
x_{i} & =\frac{(1-p) N}{k}+\sum_{j=0}^{i-2}\left(\frac{p N}{N_{\text {win }}}\right)^{j} \delta x_{1} \\
& =\frac{(1-p) N}{k}+\frac{q^{i}-q}{q^{2}-q} \delta x_{1} .
\end{aligned}
$$

The two constraints to the solution $x_{i}$ are

$$
N=\sum_{i=1}^{k} x_{i} \quad \text { and } \quad N_{\text {win }}=\sum_{i=1}^{k-1} x_{i}
$$




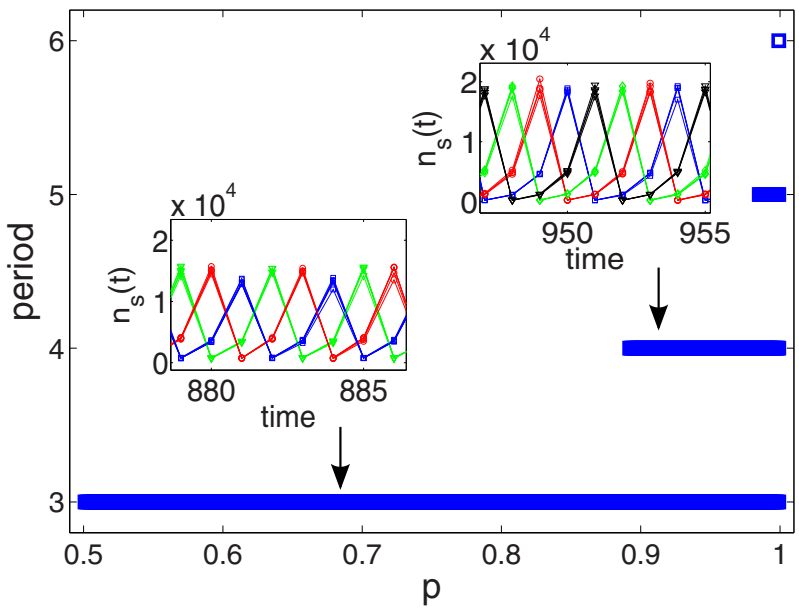

FIG. 5. (Color online) Regions of parameter $p$ in which periodic states of periods from three to six exist, obtained from Eq. (16). The insets illustrate the corresponding time series $n_{s}(t)$ of the systems at periods three and four, respectively, with the number of resources $k=16, N=10^{5}$.

which can be written, respectively, as,

$$
\begin{gathered}
N=(1-p) N+\frac{q^{k}-k q+k-1}{(1-q)^{2}} \delta x_{1}, \\
N_{\text {win }}=\frac{(1-p) N}{k}(k-1)+\frac{q^{k}-(k-1) q^{2}+(k-2) q}{(1-q)^{2} q} \delta x_{1},
\end{gathered}
$$

from which we get the simple relation

$$
\delta x_{1}=\frac{(1-p) N}{k} q .
$$

The normalized form of Eq. (14) is then

$$
1=(1-p)+\frac{q^{k}-k q+k-1}{(1-q)^{2}} \frac{q(1-p)}{k} .
$$

The emergence of the $p_{k} g_{k}$ grouping state for given $p$ value requires that the value of $q(q>0)$ obtained from Eq. (16) satisfy $x_{k-1}<N / k$, and $x_{k}>N / k$. As shown in Fig. 5, the allowable region for the solution of $p_{k} g_{k}$ grouping state decreases rapidly as $k$ is increased. It can then be concluded that periodic grouping states of high period are more and more unlikely as the period is increased, providing a plausible reason for rarity of such states in realistic systems.

\section{CONCLUSION}

A triumphant paradigm in complexity science is $\mathrm{MG}$ dynamics that are relevant to a host of phenomena in social and biological sciences [1-4]. Initially conceived through the analysis of the popular El Farol bar problem [1], the MG model with two resources has been studied extensively by researchers from various disciplines [5-28]. MG models with multiple resources were recently proposed and studied [29], where the phenomena of resource grouping and periodic oscillations were unveiled. The oscillations, however, were found to be mainly of the limit-cycle type and period two as well as its successive doubles created through period doubling like bifurcations. A question remained as to whether period-three oscillations can occur in multiple resource MG systems.

There are two main reasons to address this question. First, in nonlinear dynamical systems, period-three orbits play a fundamental role, best exemplified by the phenomenon "period three implies chaos" [30] that first defined the term "chaos" for the field. Indeed, for one-dimensional smooth maps that are representative of a host of nonlinear systems, the emergence of period-three motion immediately implies the occurrence of an infinite number of chaotic orbits (typically transient chaos). Second, triple grouping behaviors have also been observed in the U.S. housing market [31]. In particular, through a detailed analysis of the partial correlation matrix of the housing price index in each state of the United States, Zhou et al. found that the logarithmic return in the U.S. housing market reveals three clusters of comparable size in the fourth quarter in 2011. This phenomenon demands an explanation from the standpoint of complex dynamical systems. Motivated by these considerations, we derive theoretical criteria for the emergence of triple grouping states with period-three oscillations and provide solid numerical support. We also investigate the physical issue of the effect of stochastic disturbances on period-three states and identify their transient nature, with an analysis to elucidate the scaling behaviors associated with the transient lifetime.

Now that various grouping states and periodic oscillations have been uncovered in MG systems, an interesting question is whether more complicated behaviors, especially chaotic oscillations, may arise in such systems. We have not yet succeeded in identifying chaotic orbits in multiple resource MG systems. While "period three implies chaos" is well established in nonlinear dynamics, a main difficulty here is the drastic complication of the $\mathrm{MG}$ systems beyond smooth dynamical systems, as described by, for example, one-dimensional quadratic maps. It would be interesting to develop methods to search and understand potentially possible chaotic behaviors in multiple resource MG systems.

\section{ACKNOWLEDGMENTS}

Z.G.H. thanks Professor Lei Yang for helpful discussions. This work was supported by ARO under Grant No. W911NF-14-1-0504, as well as by NSF of China under Grants No. 11135001 and No. 11275003.
[1] W. B. Arthur, Am. Econ. Rev. 84, 406 (1994).

[2] D. Challet and Y.-C. Zhang, Physica A (Amsterdam, Neth.) 246, 407 (1997)
[3] H. Gintis, Game Theory Evolving: A Problem-Centered Introduction to Modeling Strategic Interaction, 2nd ed. (Princeton University Press, Princeton, NJ, 2009). 
[4] N. Johnson, P. Jefferies, and P. Hui, Financial Market Complexity (Oxford University Press, Oxford, UK, 2003).

[5] D. Challet and M. Marsili, Phys. Rev. E 60, R6271 (1999).

[6] R. Savit, R. Manuca, and R. Riolo, Phys. Rev. Lett. 82, 2203 (1999).

[7] N. F. Johnson, M. Hart, and P. M. Hui, Physica A 269, 1 (1999).

[8] D. Challet, M. Marsili, and R. Zecchina, Phys. Rev. Lett. 84, 1824 (2000).

[9] T. Kalinowski, H.-J. Schulz, and M. Birese, Physica A 277, 502 (2000).

[10] M. Paczuski, K. E. Bassler, and A. Corral, Phys. Rev. Lett. 84, 3185 (2000).

[11] F. Slanina, Physica A 299, 334 (2001).

[12] M. Hart, P. Jefferies, N. F. Johnson, and P. M. Hui, Physica A (Amsterdam, Neth.) 298, 537 (2001).

[13] A. D. Martino, M. Marsili, and R. Mulet, Europhys. Lett. 65, 283 (2004).

[14] M. Anghel, Z. Toroczkai, K. E. Bassler, and G. Korniss, Phys. Rev. Lett. 92, 058701 (2004).

[15] T. S. Lo, H. Y. Chan, P. M. Hui, and N. F. Johnson, Phys. Rev. E 70, 056102 (2004).

[16] T. Zhou, B.-H. Wang, P.-L. Zhou, C.-X. Yang, and J. Liu, Phys. Rev. E 72, 046139 (2005).

[17] T. S. Lo, K. P. Chan, P. M. Hui, and N. F. Johnson, Phys. Rev. E 71, 050101 (2005).

[18] Y.-B. Xie, B.-H. Wang, C.-K. Hu, and T. Zhou, Eur. Phys. J. B 47, 587 (2005).
[19] L.-X. Zhong, D.-F. Zheng, B. Zheng, and P. M. Hui, Phys. Rev. E 72, 026134 (2005).

[20] C. Borghesi, M. Marsili, and S. Miccichè, Phys. Rev. E 76, 026104 (2007).

[21] D. Challet, A. D. Martino, and M. Marsili, J. Stat. Mech. (2008) L04004.

[22] G. Bianconi, A. D. Martino, F. F. Ferreira, and M. Marsili, Quant. Finance 8, 225 (2008).

[23] Y. Baek, S. H. Lee, and H. Jeong, Phys. Rev. E 82, 026109 (2010).

[24] S. Biswas, A. Ghosh, A. Chatterjee, T. Naskar, and B. K. Chakrabarti, Phys. Rev. E 85, 031104 (2012).

[25] J.-Q. Zhang, Z.-G. Huang, J.-Q. Dong, L. Huang, and Y.-C. Lai, Phys. Rev. E 87, 052808 (2013).

[26] E. Moro, Advances in Condensed Matter and Statistical Physics (Nova Science, Happauge, NY, 2004).

[27] D. Challet, M. Marsili, and Y.-C. Zhang, Minority Games (Oxford University Press, Oxford, UK, 2005).

[28] C. H. Yeung and Y.-C. Zhang, in Encyclopedia of Complexity and Systems Science, edited by R. A. Meyers (Springer, New York, 2009), pp. 5588-5604.

[29] Z.-G. Huang, J.-Q. Zhang, J.-Q. Dong, L. Huang, and Y.-C. Lai, Sci. Rep. 2, 703 (2012).

[30] T. Li and J. Yorke, Am. Math. Mon. 82, 985 (1975).

[31] H. Meng, W.-J. Xie, Z.-Q. Jiang, B. Podobnik, W.-X. Zhou, and H. E. Stanley, Sci. Rep. 4, 3655 (2014).

[32] Z. Wang, B. Xu, and H.-J. Zhou, Sci. Rep. 4, 5830 (2014). 